\title{
The persistence of the attentional bias to regularities in a changing environment
}

\author{
Ru Qi Yu ${ }^{1}$. Jiaying Zhao ${ }^{1,2}$
}

Published online: 3 June 2015

(C) The Psychonomic Society, Inc. 2015

\begin{abstract}
The environment often is stable, but some aspects may change over time. The challenge for the visual system is to discover and flexibly adapt to the changes. We examined how attention is shifted in the presence of changes in the underlying structure of the environment. In six experiments, observers viewed four simultaneous streams of objects while performing a visual search task. In the first half of each experiment, the stream in the structured location contained regularities, the shapes in the random location were randomized, and gray squares appeared in two neutral locations. In the second half, the stream in the structured or the random location may change. In the first half of all experiments, visual search was facilitated in the structured location, suggesting that attention was consistently biased toward regularities. In the second half, this bias persisted in the structured location when no change occurred (Experiment 1), when the regularities were removed (Experiment 2), or when new regularities embedded in the original or novel stimuli emerged in the previously random location (Experiments 3 and 6). However, visual search was numerically but no longer reliably faster in the structured location when the initial regularities were removed and new
\end{abstract}

Electronic supplementary material The online version of this article (doi:10.3758/s13414-015-0930-5) contains supplementary material, which is available to authorized users.

Jiaying Zhao

jiayingz@psych.ubc.ca

1 Department of Psychology, University of British Columbia, Vancouver, B.C., Canada

2 Institute for Resources, Environment and Sustainability, University of British Columbia, Vancouver, B.C., Canada regularities were introduced in the previously random location (Experiment 4), or when novel random stimuli appeared in the random location (Experiment 5). This suggests that the attentional bias was weakened. Overall, the results demonstrate that the attentional bias to regularities was persistent but also sensitive to changes in the environment.

Keywords Statistical learning $\cdot$ Updating $\cdot$ Attentional control · Visual search · Primacy effect

\section{Introduction}

The environment contains a considerable number of regularities, most of which tend to remain stable over space and time. Some aspects of the environment, however, may change. For example, the shape of the shoreline may fluctuate seasonally on an otherwise stable landscape. The challenge for the visual system is to discover the emerging changes in a stable environment. This also poses a significant demand on learning. In order to form a veridical representation of the environment, the mind needs to not only extract the stable aspects of the environment, but also update the existing representation in the presence of new information.

One form of processing that supports the automatic acquisition of regularities is statistical learning, which involves the rapid extraction of statistical relationships among individual objects in space and time (Fiser \& Aslin, 2001; Saffran, Aslin, \& Newport, 1996; Turk-Browne, Jungé, \& Scholl, 2005). In a pioneering study (Saffran et al., 1996), 8-month-old infants were able to detect the reliable co-occurrences of specific syllables in a continuous auditory stream. The finding is that when three syllables (e.g., bi, da, ku) co-occurred in a triplet (e.g., bidaku) repeatedly over time, infants could distinguish 
the specific triplet order from the same syllables presented in a different order. In addition to auditory stimuli, statistical learning operates in multiple sensory modalities (Conway \& Christiansen, 2006; Fiser \& Aslin, 2001) and many feature dimensions (Chun \& Jiang, 1998; Turk-Browne, Isola, Scholl, $\&$ Treat, 2008).

Statistical learning occurs incidentally to ongoing tasks (Turk-Browne, Scholl, Johnson, \& Chun, 2010), without explicit awareness or conscious intent (Baker, Olson, \& Behrmann, 2004; Turk-Browne et al., 2005), and can interfere with ongoing tasks (Zhao, Ngo, McKendrick, \& TurkBrowne, 2011). One mechanism that allows statistical learning is attention, such that learning only occurs for attended stimuli (Campbell, Zimerman, Healey, Lee, \& Hasher, 2012; Turk-Browne et al., 2005). Moreover, attention is automatically and implicitly biased toward regularities during statistical learning, which may support further extraction of regularities (Zhao, Al-Aidroos, \& Turk-Browne, 2013). Specifically, four independent streams of objects were presented continuously and synchronously in four spatial locations around a central fixation point (Zhao et al., 2013). One stream contained a structured sequence of objects in which three objects reliably co-occurred in a specific order, similar to the syllable triplet in Saffran and colleagues' study (1996). The other three streams contained objects presented in a random order. The four steams were occasionally interrupted by a visual search array in which a target and three distractors appeared in the four locations. The target appeared in each location with equal frequency, so the structured stream was not predictive of target location. Participants viewed the four object streams, and when the search array was presented, they searched for the target in the array and discriminated its orientation. Participants were faster to detect the target when it appeared in the location containing the structured stream than in locations with random streams, even though the regularities were irrelevant to the task. The finding suggests that attention was biased toward to the spatial location containing regularities.

What remains to be understood is how the visual system updates the allocation of attention in the presence of changes of the underlying structure in the environment. Recent studies have suggested that the learning of regularities is subject to a primacy effect (Gebhart, Aslin, \& Newport, 2009; Jungé, Scholl, \& Chun, 2007; Jiang, Swallow, Rosenbaum, \& Herzig, 2013; Lew-Williams \& Saffran, 2012). For example, in one study (Gebhart et al., 2009) participants were first exposed to one artificial language containing syllables that reliably co-occurred in a specific order. They were later exposed to a second language that contained a new set of syllables that co-occurred in a different order. Participants were able to identify the co-occurring syllables in the first language, but not in the second language. This suggests that learning the regularities in the first language blocks learning of the second language. However, participants did successfully learn the second language if there was an explicit pause cue between the two languages or if the exposure to the second language was sufficiently long (Gebhart et al., 2009).

The initial exposure to regularities can prioritize the processing of the regularities (Zhao et al., 2013), even when the regularities are no longer present (Jiang et al., 2013; Jiang, Won, \& Swallow, 2014). In a probabilistic cueing study, participants detected a target which was more likely to appear in one quadrant (rich quadrant) than in other quadrants (sparse quadrants), and the detection was faster when the target appeared in the rich quadrant than in the sparse quadrants (Jiang et al., 2013). Importantly, target detection remained faster in the rich quadrant when the target was equally likely to appear in any quadrant. This suggests that attention persisted in the rich quadrant even when the quadrant was no longer predictive of the target.

The persistence of attention to the initial regularities provides an explanation of the primacy effect in statistical learning. That is, the initial exposure to regularities may prevent attention from being reallocated to new regularities, and therefore block subsequent learning of the new regularities. This raises an important question of how the attentional prioritization of regularities is updated during statistical learning when the underlying regularities change. Based on previous studies on probabilistic cueing, one prediction is that attention is persistently drawn to the initial regularities. However, in probabilistic cueing tasks, the regularities were helpful for the visual search task (i.e., the rich quadrant predicted target location). This could explain the persistence of the attentional bias to regularities. In contrast, the regularities during statistical learning were completely irrelevant to the search task (Zhao et al., 2013). Thus, it is possible that attention may not persist with the initial regularities in the context of change. In other words, the attentional bias to regularities may be flexibly updated when the regularities change.

The goal of our current study was to examine the persistence or the robustness of the attentional bias to regularities during statistical learning in the presence of new information. To directly test how attention is maintained and updated when the underlying regularities change, we revised the paradigm used in Zhao et al. (2013) and conducted six experiments. In the revised paradigm, participants viewed four simultaneous object streams in four locations on the screen, while performing an occasional visual search task. A target appeared in each location with equal probability and participants searched for the target and discriminated its orientation. The purpose of the visual search task was to gauge the allocation of attention across the four locations.

In the first half of each experiment, one stream was structured containing regularities (i.e., object triplets), one stream was random, and two streams were neutral. The neutral streams served as a baseline to examine whether attention 
was enhanced in the structured stream or suppressed in the random stream. To assess the persistence of the attentional bias to regularities, in the second half we introduced various changes to the structured stream, the random stream, or both. Specifically, as a baseline no change occurred in any stream (Experiment 1), the initial regularities were removed in the structured stream (Experiment 2), new regularities emerged in the random stream (Experiment 3), the initial regularities disappeared and new regularities emerged (Experiment 4), novel random objects appeared in the random stream (Experiment 5), or new regularities embedded in novel objects appeared in the random stream (Experiment 6). These experiments sought to characterize the persistence of the attentional bias to regularities in the presence of new information.

\section{General method}

This section described the methods common in all six experiments. In each experiment, the first half was identical. The second half differed across experiments and was described separately in each experiment.

\section{Participants}

A total of 176 undergraduate students (122 females, 54 males, mean age $=20.7$ years, standard deviation $[\mathrm{SD}]=2.9)$ from the University of British Columbia (UBC) participated in the experiments. Participants were recruited from the Human Subject Pool from the Department of Psychology and were randomly assigned to each experiment. All participants provided written informed consent. All studies reported here have been approved by the UBC Behavioral Research Ethics Board. Participants received course credit in return for their participation.

\section{Apparatus}

In all experiments, participants were seated $50 \mathrm{~cm}$ from a computer monitor (refresh rate $=60 \mathrm{~Hz}$ ). Stimuli were presented using MATLAB and the Psychophysics Toolbox.

\section{Stimuli}

In the first half of each experiment, the stimuli consisted of objects subtending $3.3^{\circ}$ on a white background presented in four streams, in the top, bottom, left, and right locations on the screen. Each location was centered $5.1^{\circ}$ from fixation, marked by a black outline. The stimuli in the streams in two opposing locations (either top and bottom, or left and right, randomly determined and remained constant for each participant) were selected from two sets of nine black shapes (Fig. 1a). In one location, the stream was structured, whereas in the opposite location the stream was random. In the structured stream, the nine shapes were randomly assigned to three triplets $(\mathrm{ABC}$, DEF, GHI). In each triplet, the three shapes always appeared in the same order. Each triplet was repeated 25 times in a pseudorandom order without back-to-back repetition of the same triplet (ABCGHIDEFABC...). In the random stream, the nine shapes appeared in a random order, and each shape was repeated 25 times without back-to-back repetition of the same shape. The streams in the remaining two locations were neutral, containing only gray squares (Figure 1a). The purpose of the neutral streams was to examine whether attention was enhanced in the structured location, or suppressed in the random location. The shapes and the gray squares appeared simultaneously and synchronously, resulting in four objects appearing in four locations per trial. This produced 225 trials $(9 \times 25)$, with the frequency of each shape equated within and between the structured and the random streams.

The four streams were interrupted occasionally and randomly by a visual search array (Figure 1b). Each array contained one target and three distractors appearing simultaneously and randomly in the four locations. The target was a " $\mathrm{T}$ " rotated $90^{\circ}$ (pointing left) or $-90^{\circ}$ (pointing right). The distractors were "L"s rotated $0^{\circ}$ (pointing right) or $180^{\circ}$ (pointing left), with the horizontal line randomly offset $20 \%$ from the bottom or top of the vertical line. This offset was to increase discrimination difficulty. The pointing directions of the target and distractors were counterbalanced in each array. That is, if the target pointed right, then two distractors pointed left and one distractor right. This ensured that any given distractor did not predict target orientation. The target appeared with equal frequency in each location. Thus, the four streams were completely irrelevant to the visual search task: there was no benefit of attending to the structured stream to find the target, and the target location was not informative about which stream was structured. Moreover, the visual search arrays were randomly interleaved into the streams, so there was no way to predict the onset of the search array, and the onset of the array was not predictable of the triplet structure.

The purpose of the search task was to gauge where attention was allocated across the four locations. For example, if discrimination was faster for targets appearing in the structured location than in the random location, this shows that the structured stream was preferentially attended compared to the random stream, suggesting that attention was drawn to the structured location. The presence of the neutral streams can further tease apart whether attention was enhanced in the structured location (indicated by faster discrimination in the structured location than in the neutral location), or suppressed 
(a) First half

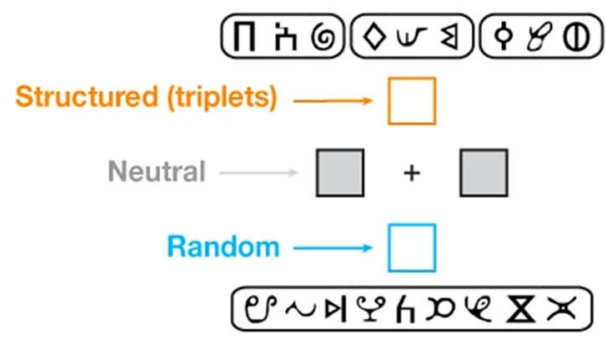

Fig. 1 General paradigm. (a) In the first half of exposure, streams of objects appeared at the top, bottom, left, and right of fixation. The stream in one location contained temporal regularities (the structured stream). The nine shapes in this stream were grouped into three triplets, whose members always appeared in the same order. The stream opposite the structured stream was random (the random stream), with the nine

in the random location (indicated by faster discrimination in the neutral location than in the random location).

It is important to emphasize that faster target discrimination reflects attentional prioritization of a location, rather than timing differences in the four streams. This is because of three reasons: First, in every trial during exposure, four objects appeared simultaneously in the four locations and disappeared simultaneously. Thus, there was no timing difference in the onset and the offset of the stimuli. Second, the search arrays were randomly interleaved into the stimulus streams, and thus there was no way to predict when the search array would appear. Finally, in each search array, one target and three distractors were randomly and simultaneously presented in the four locations. Thus, there was no way to predict where the target would appear.

\section{Procedure}

Each experiment consisted of an exposure phase that presented the four streams and visual search trials, and a test phase to assess whether participants could explicitly identify the triplets. The exposure phase consisted of two halves. The first half was identical in all six experiments. The second half differed across experiments. There was no explicit transition from the first to the second halves, and all streams appeared continuously throughout the exposure phase. Participants were not told about the two halves, or any triplets in the structured stream.

During exposure, the four objects from the four streams were presented for $750 \mathrm{~ms}$ followed by an interstimulus interval (ISI) for $750 \mathrm{~ms}$. Participants were instructed to fixate at the central cross while attending to all four streams. The occasional visual search trial was presented for $750 \mathrm{~ms}$, followed by a minimum ISI of $750 \mathrm{~ms}$. Participants indicated as accurately and quickly as possible whether the target pointed left (' 1 ' key) or right (' 0 ' key). If they did not respond during the search trial presentation or minimum ISI, the screen remained shapes appearing in a random order. The remaining two streams were neutral and contained only gray squares. (b) These four streams were occasionally interrupted by a visual search array that contained one $\mathrm{T}$ target (indicated by an arrow) and three L distractors in the four locations. The target discrimination task involved finding the target $\mathrm{T}$ and indicating whether it pointed left or right

blank until response. There were 40 search trials interspersed randomly into the 225 shape trials (none back-to-back), resulting in an average lag of 5.6 trials. In all experiments, participants were first shown five random shape trials and one search trial for practice and to clarify the instructions.

To verify that participants learned the regularities, exposure was followed by a test phase intended to measure the degree of familiarity of the regularities. In each trial, participants viewed two sets of three shapes presented at fixation and separated by a $1,000-\mathrm{ms}$ pause. Each shape appeared for $750 \mathrm{~ms}$ followed by a $750-\mathrm{ms}$ ISI. Participants judged whether the first or second set seemed more familiar. One set was a triplet from the structured stream and the other was a foil of three shapes from the same triplet. The foil was constructed by choosing one shape from each triplet (AEI, DHC, GBF). Triplets were tested against each foil twice, for a total of 18 trials (equating frequency of triplets and foils). Successful discrimination of triplets from foils depended on the knowledge of which shapes followed each other during exposure. The order of trials was randomized and whether the triplet or foil appeared first was counterbalanced across trials. After the test phase, debriefing was conducted in all experiments. Participants were asked to report whether they noticed any order in which the stimuli appeared on the screen. In all experiments, none of the participants explicitly reported seeing any order or triplets in the exposure phase, suggesting that the knowledge of the regularities was implicit.

\section{Experiment 1}

In this experiment, the second half of the exposure phase was identical to the first half. Thus, this experiment not only examined whether attention is drawn to the structured stream using this new paradigm, but also provided a baseline for the other experiments where the second half involved a change in the underlying regularities. 


\section{Stimuli and procedure}

The first half of the exposure phase was described in General Methods above. The second half was identical to the first half (Figure 2a). In all experiments, there was no explicit transition between the two halves, and all streams appeared continuously throughout the exposure phase. Participants $(\mathrm{N}=22)$ completed the exposure phase and the test phase described above.

\section{Results and discussion}

At the test phase, triplets were chosen over foils on 59.8\% (SD $=18.2 \%$ ) of the time, revealing statistical learning [chance $=$ $50 \% ; t(21)=2.54, p=0.02, d=0.54]$. This shows that in this new paradigm temporal regularities can be learned at one location despite the random and neutral stimuli appearing in the other locations.

The accuracy of the target discrimination task during visual search trials was $79.7 \%(\mathrm{SD}=8.0 \%)$, and there was no reliable difference among the structured, random, and neutral locations via a one-way ANOVA $[F(2,42)=1.26, p=0.29$, $\left.\eta_{p}{ }^{2}=0.06\right]$. Thus, we used the response times (RTs) of only correct trials. RTs greater than 3SDs from the mean in each condition were trimmed (1.6\% of all trials). The accuracy and inverse efficiency (RT divided by accuracy; Kristjánsson, Sigurjónsdóttir, \& Driver, 2010; Townsend \& Ashby, 1983) results for each location and each half of all experiments were attached in the Supplementary Material.

Target discrimination RTs were analyzed with a 3 (location: structured, random, neutral) $\times 2$ (time: first half, second half) repeated-measures ANOVA. There was a main effect of location $\left[F(2,42)=6.71, p=0.003, \eta_{p}{ }^{2}=0.24\right]$ but no main effect of time $\left[F(1,21)=0.24, p=0.63, \eta_{p}{ }^{2}=0.01\right]$ or interaction $\left[F(2,42)=1.31, p=0.28, \eta_{p}{ }^{2}=0.06\right]$. Planned comparisons revealed that RTs were reliably faster for targets in the structured location than in the random location in the first half ${ }^{1}$ $[t(21)=2.18, p=0.04, d=0.46]$ and also in the second half $[t(21)=2.55, p=0.02, d=0.54]$ of the exposure phase (Figure 2a). RTs also were faster in the structured location than in the neutral locations in the first half $[t(21)=2.87, p=0.01$, $d=0.61]$ and in the second half $[t(21)=2.93, p=0.008, d=$ $0.63]$ of exposure. There was no reliable difference in RTs between the random and the neutral locations in both halves $\left[t^{\prime} \mathrm{s}<1.5, p\right.$ 's $\left.>0.15, d^{\prime} \mathrm{s}<0.30\right]$. This suggests that attention is enhanced in the structured location, rather than suppressed in the random location.

Although the shapes were completely task irrelevant, targets appearing in the location of the structured stream were

\footnotetext{
${ }^{1}$ The RT benefit in the structured location was not statistically different when the structured location was on the top, bottom, left, or right side of fixation $\left[F(3)=1.19, p=0.31, \eta_{p}{ }^{2}=0.02\right]$.
}

preferentially attended compared with targets in the random or neutral locations. This suggests that attention is enhanced at the location of temporal regularities. This baseline replicated the findings in Zhao et al. (2013) and validated the current paradigm.

\section{Experiment 2}

The goal of this experiment was to examine how persistent the attentional bias is in the presence of a change in the underlying regularities. Specifically, the change involved the removal of regularities in the previously structured stream.

\section{Stimuli and procedure}

The stimuli were identical to those in Experiment 1, except that in the second half of the exposure phase, the stimuli in the structured stream now appeared in a random order. In other words, there were two random streams in the second half (Figure 2b). Participants $(\mathrm{N}=$ 22) took part in this experiment.

\section{Results and discussion}

Triplets were chosen over foils on $55.6 \%$ ( $\mathrm{SD}=12.4 \%)$ of test trials, which was reliably above chance $[t(21)=2.11, p<0.05$, $d=0.12]$. This shows that participants were still able to recognize the triplets despite the fact that regularities were removed in the second half of the experiment.

The accuracy of the target discrimination task was $83.2 \%(\mathrm{SD}=6.9 \%)$, and there was no reliable difference among the locations via a one-way ANOVA (locations: structured, random, neutral) $[F(2,42)=0.61, p=$ $\left.0.55, \eta_{p}{ }^{2}=0.03\right]$. Thus, we used the RTs of the correct trials. RTs greater than $3 \mathrm{SDs}$ from the mean were trimmed ( $1.4 \%$ of all trials).

Target discrimination RTs were analyzed with a 2 (experiment: 1, 2) $\times 3$ (location: structured, random, neutral) $\times 2$ (time: first half, second half) mixed-effects ANOVA (between-subject: experiment; within-subject: location and time). There was a main effect of location $[F(2,84)=9.90, p<0.001$, $\left.\eta_{p}{ }^{2}=0.19\right]$ but no main effect of experiment $[F(1,42)=0.15, p$ $\left.=0.70, \eta_{p}{ }^{2}=0.003\right]$, time $\left[F(1,42)=1.10, p=0.30, \eta_{p}{ }^{2}=\right.$ $0.03]$, or interaction $\left[F(2,84)=1.53, p=0.22, \eta_{p}{ }^{2}=0.04\right]$. This suggests that the results of this experiment were not statistically different from those in Experiment 1 where no change occurred.

A further 3 (location: structured, random, neutral) $\times$ 2 (time: first half, second half) ANOVA revealed that 


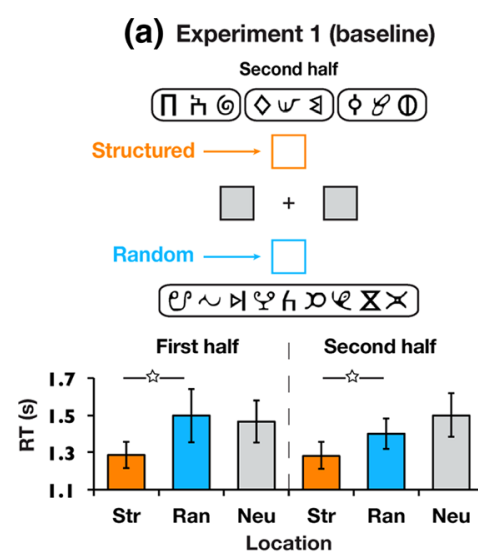

(d) Experiment 4

Second half

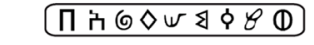

Random $\longrightarrow$

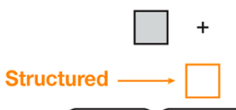

er

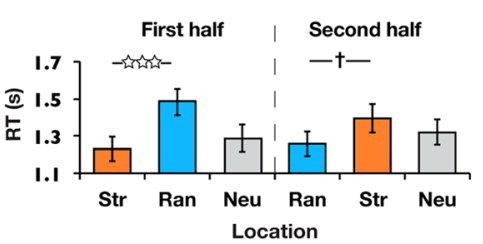

Fig. 2 Results. (a) Experiment 1. This served as a baseline where the second half of the exposure phase was identical to the first half. The graph shows mean response time in the visual search task as a function of whether the target appeared at the location of the structured stream, the random stream, or the neutral streams. (b) Experiment 2. In the second half of exposure, the stimuli in the previously structured stream appeared in a random order. (c) Experiment 3. In the second half, the stimuli in the previously random stream appeared in triplets. (d) Experiment 4 . In the

there was a main effect of location $[F(2,42)=3.50, p$ $\left.=0.04, \eta_{p}{ }^{2}=0.14\right]$ but no main effect of time $[F(1,21)$ $\left.=1.09, p=0.31, \eta_{p}{ }^{2}=0.05\right]$ or interaction $[F(2,42)=$ $\left.0.40, p=0.68, \eta_{p}{ }^{2}=0.02\right]$. Moreover, RTs were reliably faster in the structured location than in the random location in the first half $[t(21)=2.66, p=0.01, d$ $=0.57]$ of exposure. More importantly, RTs also were reliably faster in the newly random location than in the initially random location in the second half $[t(21)=$ 2.21, $p=0.04, d=0.47$ ] (Figure 2b). RTs also were faster in the structured location than in the neutral locations in the first half $[t(21)=2.15, p=0.04, d=$ $0.46]$ but not between the newly random location and the initially random location in the second half $[t(21)=$ $1.47, p=0.15, d=0.31]$. There was no reliable difference in the RTs between random and neutral locations for both halves $[t$ 's $(21)<0.5, p$ 's $>0.60$, $d$ 's $<$ $0.10]$. Thus, attention seems to be enhanced in the structured location, rather than suppressed in the random location. (c) Experiment 3 Second half Second half

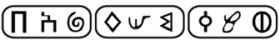

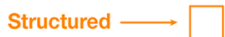

Structured $\longrightarrow \square$
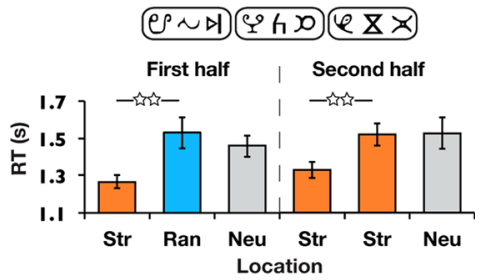

(f) Experiment 6

Experiment 5 Second half

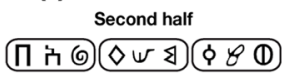
Structured $\longrightarrow \square$
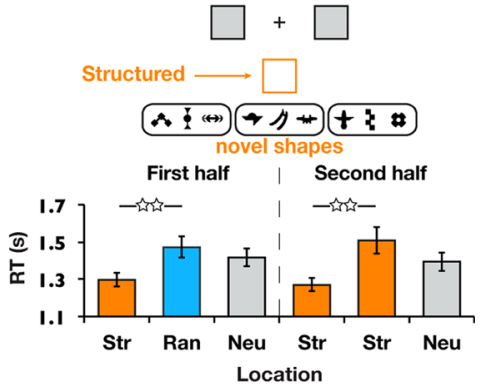

second half, the stimuli in the previously structured stream appeared in a random order, and the stimuli in the previously random stream appeared in triplets. (e) Experiment 5. In the second half, the stimuli in the previously random stream were replaced by novel shapes that also appeared in a random order. (f) Experiment 6. In the second half, the stimuli in the previously random stream were replaced by novel shapes that appeared in triplets. Error bars indicate \pm 1 between-subjects SEM $\left({ }^{*} p\right.$ $\left.<0.05, * * p<0.01, * * * p<0.001,{ }^{\dagger} p<0.1\right)$

These results suggest that attention was drawn to temporal regularities in the first half of exposure and persisted in the same location even after the regularities were removed.

\section{Experiment 3}

An alternative explanation of the persistence effect in Experiment 2 is that a change in the underlying regularities in the structured location was surprising and therefore held attention in that location. This means that rather than persisting in the same location, attention was drawn to the location because of the change in the statistical relationships among the stimuli. To address this alternative account, new regularities were introduced in the previously random stream and we examined whether attention shifted to the newly structured location. If the shift occurs, this suggests that a change in the statistical relationships in the stimuli can draw attention, discounting the persistence effect. 


\section{Stimuli and procedure}

The stimuli were identical to those in Experiment 1, except that in the second half of the exposure phase, the stimuli in the random stream now appeared in triplets, as in the structured stream. In other words, there were two structured streams in the second half (Figure 2c). Participants $(\mathrm{N}=44)$ completed the exposure phase and the test phase for the initial triplets. Among those, 22 participants also completed the test phase for the newly introduced triplets. ${ }^{2}$

\section{Results and discussion}

Triplets were chosen over foils on $56.7 \%$ (SD $=12.1 \%$ ) of test trials, which was reliably above chance $[t(43)=3.66, p<$ $0.001, d=0.55]$, revealing robust learning of the regularities in the initially structured stream. The newly formed triplets in the second half were chosen over foils on $51.7 \%$ (SD = $12.9 \%$ ) of test trials, which was not reliably above chance $[t(21)=0.64, p=0.53, d=0.14]$, suggesting that the new regularities were not learned.

The accuracy of the target discrimination task was $80.6 \%$ $(\mathrm{SD}=8.0 \%)$. There was a reliable difference among the locations via a one-way ANOVA $\left[F(2,86)=3.24, p=0.04, \eta_{p}{ }^{2}=\right.$ $0.07]$. The accuracy in the random location was lower $(77.3 \%)$ than that in the structured location $(82.2 \%)[t(43)=2.27, p=$ $0.03, d=0.34]$, and also the neutral locations $(81.5 \%)[t(43)=$ $2.01, p=0.05, d=0.30]$. This suggests that the faster RTs in the structured location, if found, were not driven by the speedaccuracy tradeoff. RTs of the correct trials were used, and those greater than $3 \mathrm{SDs}$ from the mean were trimmed $(1.8 \%$ of all trials).

Target discrimination RTs were analyzed with a 2 (experiment: 1,3$) \times 3$ (location: structured, random, neutral) $\times 2$ (time: first half, second half) mixed-effects ANOVA (between-subject: experiment; within-subject: location and time). There was a main effect of location $[F(2,128)=11.81, p<$ $\left.0.001, \eta_{p}{ }^{2}=0.16\right]$ but no main effect of experiment $[F(1,64)=$ $\left.0.14, p=0.71, \eta_{p}{ }^{2}=0.002\right]$, time $\left[F(1,64)=0.48, p=0.49, \eta_{p}{ }^{2}\right.$ $=0.008]$, or interaction $\left[F(2,128)=0.13, p=0.88, \eta_{p}{ }^{2}=\right.$ 0.002]. This suggests that the results of this experiment were not statistically different from those in Experiment 1 where no change occurred.

A further 3 (location: structured, random, neutral) $\times 2$ (time: first half, second half) ANOVA revealed that there was a main effect of location $\left[F(2,86)=7.03, p=0.001, \eta_{p}{ }^{2}\right.$ $=0.14]$ but no main effect of time $[F(1,43)=1.13, p=0.29$,

\footnotetext{
2 The original Experiment 3 contained 22 participants who did not complete the test phase for the newly introduced triplets. As suggested by two reviewers, we collected additional 22 participants who completed the test phase. The same holds for Experiment 6 .
}

$\left.\eta_{p}{ }^{2}=0.03\right]$ or interaction $\left[F(2,86)=0.91, p=0.41, \eta_{p}{ }^{2}=\right.$ $0.02]$. Moreover, RTs were reliably faster in the structured location than in the random location in the first half $[t(43)=$ $3.31, p=0.002, d=0.50]$. RTs also were reliably faster in the initially structured location than in the newly structured location in the second half $[t(43)=3.20, p=0.003, d=0.48]$ (Figure 2c). Moreover, RTs were faster in the structured location than in the neutral locations in the first half $[t(43)=3.39, p$ $=0.001, d=0.51]$ and in the second half $[t(43)=2.13, p=$ $0.04, d=0.32]$. There was no reliable difference in the RTs between random (or the newly structured location) and neutral locations $[t$ 's $(43)<1.2, p$ 's $<0.23, d$ 's $<0.19]$. Thus, attention seems to be enhanced in the structured location, rather than suppressed in the random location.

These results suggest that the newly formed regularities failed to capture attention, and attention was consistently drawn to the initially structured location throughout exposure. Thus, the persistence effect that was observed both in the current experiment and in Experiment 2 was unlikely to be driven by a mere change in the statistical relationships in the stimuli.

\section{Experiment 4}

The first three experiments revealed a persistent attentional bias toward the initially structured location, even though the regularities disappeared (Experiment 2) or new regularities were introduced in a different location (Experiment 3 ) in the second half of the experiment. A remaining possibility for testing the persistence of the bias is to introduce both changes simultaneously and observe whether the combined perturbations to the stimuli shift attention away from the initially structured location. ${ }^{3}$

\section{Stimuli and procedure}

The stimuli were identical to those in Experiment 1, except that in the second half of the exposure phase, the stimuli in the random stream now appeared in triplets, and the stimuli in the structured stream now appeared in a random order (Figure 2d). Participants $(\mathrm{N}=22)$ took part in this experiment.

\section{Results and discussion}

Triplets were chosen over foils on $55.8 \%$ ( $\mathrm{SD}=11.9 \%)$ of test trials, which was reliably above chance $[t(21)=2.28, p=0.03$, $d=0.49$ ], revealing robust learning of the regularities in the

\footnotetext{
${ }^{3}$ We thank Justin Jungé and an anonymous reviewer for raising the idea for this experiment.
} 
initially structured stream. The newly formed triplets in the second half were chosen over foils on $53.0 \%(\mathrm{SD}=9.8 \%)$ of test trials, which was not reliably above chance $[t(21)=$ $1.45, p=0.16, d=0.31$ ], suggesting that the new regularities were not learned.

The accuracy of the target discrimination task during visual search trials was $79.0 \%(\mathrm{SD}=9.9 \%)$, and there was no reliable difference among the structured, random, and neutral locations via a one-way ANOVA $[F(2,42)=1.18, p=0.32$, $\left.\eta_{p}{ }^{2}=0.05\right]$. Thus, we used the RTs of correct trials. RTs greater than $3 \mathrm{SDs}$ from the mean in each condition were trimmed (1.7\% of all trials).

Target discrimination RTs were analyzed with a 2 (experiment: 1,4$) \times 3$ (location: structured, random, neutral) $\times 2$ (time: first half, second half) mixed-effects ANOVA (between-subject: experiment; within-subject: location and time). There was a main effect of location $[F(2,84)=12.18, p<$ $\left.0.001, \eta_{p}{ }^{2}=0.22\right]$ but no main effect of experiment $[F(1,42)$ $\left.=0.48, p=0.49, \eta_{p}{ }^{2}=0.01\right]$, time $[F(1,42)=0.23, p=0.64$, $\left.\eta_{p}{ }^{2}=0.005\right]$, or interaction $\left[F(2,84)=0.04, p=0.96, \eta_{p}{ }^{2}=\right.$ $0.001]$. This suggests that the results of this experiment were not statistically different from those in Experiment 1 where no change occurred.

A further 3 (location: structured, random, neutral) $\times 2$ (time: first half, second half) ANOVA revealed that there was a main effect of location $\left[F(2,42)=8.93, p<0.001, \eta_{p}{ }^{2}\right.$ $=0.30]$ but no main effect of time $[F(1,21)=0.05, p=0.83$, $\left.\eta_{p}{ }^{2}=0.002\right]$ or interaction $\left[F(2,42)=1.80, p=0.18, \eta_{p}{ }^{2}=\right.$ $0.08]$. Moreover, RTs were reliably faster in the structured location than in the random location in the first half $[t(21)=$ $4.29, p<0.001, d=0.91]$. However, RTs were not reliably faster in the previously structured location than in the previously random location in the second half $[t(21)=1.90, p=$ $0.07, d=0.40$ ] (Figure 2d). No other pair-wise comparisons were reliable $[t$ 's $(21)<1.5, p$ 's $<0.14, d$ 's $<0.32]$.

These results suggest that attention was initially drawn to the structured location, but the bias was weakened in the second half of exposure, as indicated by the lack of the reliable difference in RT between the previously structured location and the previously random location. The weakening of the bias could reflect the competition for attention from the newly formed regularities in the previously random location, coupled with the disappearance of the initial regularities in the previously structured location.

\section{Experiment 5}

One barrier to processing the newly introduced regularities is that participants may become habituated to the noise in the random stream in the first half of exposure and block learning of new regularities in these stimuli later (Jungé et al., 2007; Gebhart et al., 2009). Such habituation is driven by two factors: the initial exposure to the randomness of the stimuli in the random stream, or the fact that the same stimuli were presented throughout exposure. To tease these two factors apart, in this experiment novel shapes were introduced in the random stream in the second half of exposure. The goal was to examine whether attention still persists in the structured location in the presence of novel objects in another location.

\section{Stimuli and procedure}

The stimuli were identical to those in Experiment 1, except that in the second half of the exposure phase, the stimuli in the random stream were replaced by a new set of shapes appearing in a random order (Figure 2e). Participants $(\mathrm{N}=$ 22) took part in this experiment.

\section{Results and discussion}

Triplets were chosen over foils on $58.8 \%$ ( $\mathrm{SD}=16.5 \%$ ) of test trials, which was reliably above chance $[t(21)=2.51, p=0.02$, $d=0.54]$, revealing robust learning of the regularities in the structured stream.

The accuracy of the target discrimination task was $78.2 \%$ $(\mathrm{SD}=9.7 \%)$, and there was no reliable difference among the locations via a one-way ANOVA $[F(2,42)=0.08, p=0.92$, $\left.\eta_{p}{ }^{2}=0.004\right]$. Thus, we used the RTs of the correct trials. RTs greater than $3 \mathrm{SDs}$ from the mean were trimmed $(1.4 \%$ of all trials).

Target discrimination RTs were analyzed with a 2 (experiment: 1,5$) \times 3$ (location: structured, random, neutral) $\times 2$ (time: first half, second half) mixed-effects ANOVA (between-subject: experiment; within-subject: location and time). There was a main effect of location $[F(2,84)=7.29, p=0.001$, $\left.\eta_{p}{ }^{2}=0.15\right]$ but no main effect of experiment $[F(1,42)=0.21, p$ $\left.=0.65, \eta_{p}{ }^{2}=0.005\right]$, time $\left[F(1,42)=0.12, p=0.73, \eta_{p}{ }^{2}=\right.$ $0.003]$, or interaction $\left[F(2,84)=1.02, p=0.37, \eta_{p}{ }^{2}=0.02\right]$. This suggests that the results of this experiment were not statistically different from those in Experiment 1 where no change occurred.

A further 3 (location: structured, random, neutral) $\times 2$ (time: first half, second half) ANOVA showed that there was a marginal effect of location $\left[F(2,42)=2.54, p=0.09, \eta_{p}{ }^{2}=\right.$ $0.11]$ but no effect of time $\left[F(1,21)=0.004, p=0.95, \eta_{p}{ }^{2}<\right.$ $0.001]$ or interaction $\left[F(2,42)=0.24, p=0.79, \eta_{p}{ }^{2}=0.01\right]$. RTs were reliably faster in the structured location than in the random location in the first half $[t(21)=2.84, p=0.01, d=$ $0.60]$ but not in the second half $[t(21)=1.42, p=0.17, d=$ 0.30 ] (Figure 2e). No other pair-wise comparisons were reliable $[t$ 's $(21)<1.92, p$ 's $<0.06, d$ 's $<0.41]$.

These results suggest that attention was initially biased toward the structured location, but the bias was weakened in the 
second half of exposure when novel random shapes were introduced in the random location.

\section{Experiment 6}

The weakening of the attentional bias to regularities in Experiment 5 suggests that novel objects can be detected during the second half of exposure. This raises the possibility that even after the initial exposure to noise, learning of novel objects may occur later on. Thus, in the final experiment novel shapes were introduced in the random location in the second half of exposure, as in Experiment 5. More importantly, these novel shapes now appeared in triplets in the previously random location. With the change of stimuli and structure, we examined the additive effect of novel shapes and new regularities on attention. The prediction is that the appearance of novel objects draws attention and shifts the bias away from the initially structured location. However, we have found that introducing new regularities in the random location did not shift the attentional bias (Experiment 3). Therefore, a competing prediction is that introducing new regularities in novel shapes does not alter the attentional bias. To determine which prediction holds true, the final experiment examined whether the attentional bias was weakened in the presence of new regularities embedded in novel shapes appearing in the previously random location.

\section{Stimuli and procedure}

The stimuli were identical to those in Experiment 5, except that in the second half of the exposure phase, the novel shapes now appeared in triplets, as in the structured stream. In other words, there were two structured streams in the second half (Figure 2f). Participants $(\mathrm{N}=44)$ completed the exposure phase and the test phase for the initial triplets. Among those, 22 participants also completed the test phase for the newly introduced triplets.

\section{Results and discussion}

Triplets were chosen over foils on $58.0 \%(\mathrm{SD}=14.8 \%)$ of test trials, which was reliably above chance $[t(43)=3.56, p<$ $0.001, d=0.54]$, revealing robust learning of the regularities in the initially structured stream. The newly formed triplets in the second half were chosen over foils on $49.2 \%$ (SD $=$ $13.1 \%$ ) of test trials, which was not different from chance $[t(21)=0.27, p=0.79, d=0.06]$, suggesting that the new regularities were not learned.

The accuracy of the target discrimination task was $82.3 \%$ $(\mathrm{SD}=6.8 \%)$, and there was no reliable difference among the locations via a one-way ANOVA $[F(2,86)=0.85, p=0.43$, $\left.\eta_{p}{ }^{2}=0.02\right]$. Thus, we used the RTs of the correct trials. RTs greater than 3 SDs from the mean were trimmed $(1.8 \%$ of all trials).

Target discrimination RTs were analyzed with a 2 (experiment: 1, 6) $\times 3$ (location: structured, random, neutral) $\times 2$ (time: first half, second half) mixed-effects ANOVA (between-subject: experiment; within-subject: location and time). There was a main effect of location $[F(2,128)=13.35, p<$ $\left.0.001, \eta_{p}{ }^{2}=0.17\right]$, but no main effect of experiment $[F(1,64)=$ $\left.0.02, p=0.89, \eta_{p}{ }^{2}<0.001\right]$, time $\left[F(1,64)=0.14, p=0.71, \eta_{p}{ }^{2}\right.$ $=0.002]$, or interaction $\left[F(2,128)=1.53, p=0.22, \eta_{p}{ }^{2}=0.02\right]$. Comparing the current results to those in Experiment 5, there was a main effect of location $[F(2,128)=10.73, p$ $\left.<0.001, \eta_{p}{ }^{2}=0.14\right]$ but no main effect of experiment $\left[F(1,64)=0.40, p=0.53, \eta_{p}{ }^{2}=0.006\right]$, time $[F(1,64)=$ $\left.0.02, p=0.89, \eta_{p}{ }^{2}<0.001\right]$ or interaction $[F(2,128)=$ 1.53, $\left.p=0.22, \eta_{p}{ }^{2}=0.02\right]$. This suggests that the results of this experiment were not statistically different from those in Experiment 1 or Experiment 5.

A further 3 (location: structured, random, neutral) $\times 2$ (time: first half, second half) ANOVA showed that there was an effect of location $\left[F(2,86)=8.37, p<0.001, \eta_{p}{ }^{2}=0.16\right]$ but no main effect of time $\left[F(1,43)=0.02, p=0.89, \eta_{p}{ }^{2}<0.001\right]$ or interaction $\left[F(2,86)=0.46, p=0.63, \eta_{p}{ }^{2}=0.01\right]$. RTs were reliably faster in the structured location than in the random location in the first half $[t(43)=3.13, p=$ $0.003, d=0.47]$. RTs also were reliably faster in the initially structured location than in the newly structured location in the second half $[t(43)=3.02, p=0.004, d=$ 0.46] (Figure 2f). Moreover, RTs were faster in the structured location than in the neutral locations in the first half $[t(43)=2.18, p=0.04, d=0.33]$ and in the second half $[t(43)=2.20, p=0.03, d=0.33]$. There was no reliable difference in the RTs between random (or the newly structured location) and neutral locations [t's $(43)<1.8, p$ 's $<0.09$, d's $<0.26]$. Thus, attention seems to be enhanced in the structured location, rather than suppressed in the random location.

These results suggest that attention was consistently drawn to the temporal regularities in the structured stream throughout exposure. Critically, new regularities in novel shapes emerging in the second half of exposure failed to capture attention. This finding is inconsistent with that in Experiment 5 where the attentional bias to the initially structured location was weakened after novel shapes appeared in the random location. However, the current finding is consistent with that in Experiment 3, where newly introduced regularities did not shift attention away from the initially structured location. The current results again show that the newly introduced regularities fail to draw attention, even though the regularities appear in novel shapes. 


\section{General discussion}

Our goal was to examine the persistence of the attentional bias to regularities in the presence of changes in the underlying structure of the stimuli. Across six experiments, visual search was consistently facilitated in the spatial location containing temporal regularities in the first half of the exposure phase. This suggests that attention was initially biased toward regularities. In all experiments, none of the participants explicitly noticed the regularities, suggesting that the attentional bias to regularities was implicit. This bias persisted in the structured location in the second half of exposure when no change occurred (Experiment 1), when the regularities were removed (Experiment 2), or when new regularities embedded in the original or novel stimuli emerged in the previously random location (Experiments 3 and 6). The facilitation of visual search in the structured location was driven by an enhancement of attention to the structured stream, rather than the suppression of the random location.

One reason of the persistence effect is to ensure the successful learning of the regularities upon initial exposure. Because the stimuli in the initial structured stream were complex (i.e., each triplet contained three arbitrary objects presented in a fixed order, and the stream contained three triplets presented in a random order), knowledge of the regularities might be imperfect given the duration of exposure in the experiment. Indeed, the performance of the test phase was never above $60 \%$ across our experiments. Such imperfect knowledge may drive attention to the structured stream to allow more learning to occur in the second half of the experiment. In other words, the processing of regularities was enhanced through implicit attentional control mechanisms when new information was presented in a different location. This enhancement can be facilitated by a close-loop interaction between learning and attention, in that more selective attention allows for more learning and more learning results in a stronger attentional focus. Thus, attention persisted in the structured location in order to allow the exploitation of regularities that had not been sufficiently acquired.

The pattern of results across all six experiments was similar, and there was no interaction between any experiment and the baseline Experiment 1. Nonetheless, in Experiments 4 and 5 the RT difference between the previously structured and random locations was no longer statistically significant in the second half of exposure, but the numerical difference still remained. While this result does not necessarily indicate the dissipation of the attentional bias, the lack of statistical difference reflects the weakening of the attentional bias to the previously structured location in the second half. Thus, the attentional bias to regularities was reduced when the initial regularities were removed in the structured location and new regularities were introduced in the previously random location (Experiment 4) or when novel random shapes appeared in the random location (Experiment 5). This suggests that the attentional bias to regularities persisted in our experiments, but it was sensitive to certain environmental changes.

The findings from the six experiments revealed two interesting aspects of the persistence of the attentional bias to regularities in the context of change. First, neither removing regularities in the initially structured location (Experiment 2), nor introducing new regularities in the initially random location (Experiment 3) influenced the attentional bias to regularities, but the combination of the two changes (Experiment 4) weakened the bias. Second, neither the presence of novel stimuli (Experiment 6), nor the continued presence of random stimuli in the random location (Experiment 1) influenced the attentional bias, but the combination of the two (Experiment 5) weakened the bias.

The persistence of the bias in Experiments 3 and 6 offers insights on how multiple sources of structure are prioritized for processing during statistical learning. That is, when two sources of regularities are present, the initial encounter of regularities in one location may have blocked learning of subsequent new regularities in a different location. Any information irrelevant to the initial regularities may be blocked for processing through selective attention (Broadbent, 1958; Conway, Cowan, \& Bunting, 2001). This is consistent with previous studies on statistical learning that also demonstrate the blocking of subsequent new information by the exposure to the initial structure (Gebhart et al., 2009; Weiss, Gerfen, \& Mitchel, 2009). Participants in Experiments 3 and 6 failed to learn the new regularities based on their performance in the test phase. This is not surprising, because these new regularities were never prioritized in the second half of exposure. However, learning of the new regularities may still be possible if the exposure is extended (Gebhart et al., 2009). This also suggests that statistical learning may proceed in a serial manner from one structured stream to another, and regularities in a different stream will only be processed when the initial regularities have been learned.

The weakening of the bias in Experiment 4 suggests detection of new regularities is possible if the original regularities in the structured location were no longer present. The removal of the initial regularities thus seems critical to the acquisition of subsequent new regularities. However, the removal of regularities alone was not sufficient to weaken the attentional bias to the initial regularities (Experiment 2), and in fact the bias was equally strong in both halves of exposure. This persistence effect is consistent with previous studies on probabilistic cueing which showed that attention was persistently drawn to a spatial location in which targets were initially more likely to appear (Jiang et al., 2013; Jiang et al., 2014). The removal of the initial regularities coupled with the addition of new regularities reduced the attentional bias, but the bias had not reversed. That is, the RT in the visual search task was still numerically lower when targets appeared in the initially 
structured location than in the initially random location. It remains to be tested whether extending the exposure to the newly introduced regularities would eventually shift attention from the initial regularities to the new regularities, and result in the successful learning of the new regularities.

The weakening of the attentional bias to regularities in Experiment 5 suggests that the presence of novel random stimuli can capture attention, even though stimulus novelty alone (Experiment 6) or randomness alone (Experiment 1) had no impact on the attentional bias. The result in Experiment 5 is inconsistent with that in Experiment 6, where novel structured stimuli failed to shift the attentional bias. One explanation for this inconsistency involves the rapid detection of regularities. In Experiment 6, when the visual system encounters the novel shapes in the second half of exposure, it may have quickly detected some reliable co-occurrences between the novel shapes. Such detection may trigger the implicit blocking of further processing of the new regularities, allowing attention to return to the initial regularities. However, in Experiment 5 because the novel shapes are random (lacking regularities), this blocking mechanism may not be triggered, and as a result attention is drawn to the novel shapes (Johnston et al., 1990). If this is true, then with sufficient exposure to novel random shapes attention may eventually return to regularities in the structured location. More studies are needed to examine this account.

In our experiments, the changes in the underlying regularities in the stimulus stream included not only an alteration of the order in which the shapes appeared, but also a complete replacement with new objects. Future investigation is needed to identify other circumstances under which the attentional bias to regularities shifts. For example, the bias may dissipate after extensive exposure to regularities in the absence of change. In fact, Zhao \& Turk-Browne (in preparation) conducted a study in which the duration of the Experiment 1 was tripled. They found that attention was initially biased toward the structured location in the first epoch of the experiment, but in the third epoch attention gradually shifted to the random location. This suggests that the attentional bias for regularities is transient over a longer timescale.

\section{Conclusions}

We found that attention was initially drawn to regularities, and this bias persisted when no change occurred, when the regularities were removed, or when new regularities emerged. However, the attentional bias was weakened when the initial regularities were removed and new regularities were introduced, or when novel random stimuli were presented. Overall, the results suggest that the attentional bias to regularities was persistent but also sensitive to changes in the information in the environment. The current finding provides new insights into how statistical learning is maintained and updated in a changing environment.

Acknowledgments For helpful conversations, the authors thank Nicholas B. Turk-Browne, Richard Aslin, József Fiser, James Enns, Justin Jungé, and the Zhao Lab. The authors thank Yu Luo and James Jiang for assistance with data collection. This work was supported by NSERC Discovery Grant RGPIN-2014-05617 (to JZ).

\section{References}

Baker, C. I., Olson, C. R., \& Behrmann, M. (2004). Role of attention and perceptual grouping in visual statistical learning. Psychological Science, 15, 460-466.

Broadbent, D. E. (1958). Perception and communication. New York: Oxford University Press.

Campbell, K. L., Zimerman, S., Healey, M. K., Lee, M., \& Hasher, L. (2012). Age differences in visual statistical learning. Psychology and Aging, 27, 650-656.

Chun, M. M., \& Jiang, Y. (1998). Contextual cueing: Implicit learning and memory of visual context guides spatial attention. Cognitive Psychology, 36, 28-71.

Conway, C. M., \& Christiansen, M. H. (2006). Statistical learning within and between modalities pitting abstract against stimulus-specific representations. Psychological Science, 17, 905-912.

Conway, A. R., Cowan, N., \& Bunting, M. F. (2001). The cocktail party phenomenon revisited: The importance of working memory capacity. Psychonomic Bulletin \& Review, 8, 331-335.

Fiser, J., \& Aslin, R. N. (2001). Unsupervised statistical learning of higher-order spatial structures from visual scenes. Psychological Science, 12, 499-504.

Gebhart, A. L., Aslin, R. N., \& Newport, E. L. (2009). Changing structures in midstream: Learning along the statistical garden path. Cognitive Science, 33, 1087-1116.

Jiang, Y. V., Swallow, K. M., Rosenbaum, G. M., \& Herzig, C. (2013). Rapid acquisition but slow extinction of an attentional bias in space. Journal of Experimental Psychology: Human Perception and Performance, 39, 87-99.

Jiang, Y. V., Won, B. Y., \& Swallow, K. M. (2014). First saccadic eye movement reveals persistent attentional guidance by implicit learning. Journal of Experimental Psychology: Human Perception and Performance, 40, 1161-1173.

Johnston, W. A., Hawley, K. J., Plew, S. H., Elliott, J. M., \& DeWitt, M. J. (1990). Attention capture by novel stimuli. Journal of Experimental Psychology: General, 119, 397-411.

Jungé, J. A., Scholl, B. J., \& Chun, M. M. (2007). How is spatial context learning integrated over signal versus noise? A primacy effect in contextual cueing. Visual Cognition, 15, 1-11.

Kristjánsson, Á., Sigurjónsdóttir, Ó., \& Driver, J. (2010). Fortune and reversals of fortune in visual search: Reward contingencies for pop-out targets affect search efficiency and target repetition effects. Attention, Perception, \& Psychophysics, 72(5), 1229-1236.

Lew-Williams, C., \& Saffran, J. R. (2012). All words are not created equal: Expectations about word length guide infant statistical learning. Cognition, 122, 241-246.

Saffran, J. R., Aslin, R. N., \& Newport, E. L. (1996). Statistical learning by 8-month-old infants. Science, 274, 1926-1928.

Townsend, J. T., \& Ashby, F. G. (1983). The Stochastic Modelling of Elementary Psychological Processes. Cambridge: Cambridge University Press.

Turk-Browne, N. B., Jungé, J. A., \& Scholl, B. J. (2005). The automaticity of visual statistical learning. Journal of Experimental Psychology: General, 134, 552-564. 
Turk-Browne, N. B., Isola, P. J., Scholl, B. J., \& Treat, T. A. (2008). Multidimensional visual statistical learning. Journal of Experimental Psychology: Learning, Memory, and Cognition, 34, 399-407.

Turk-Browne, N. B., Scholl, B. J., Johnson, M. K., \& Chun, M. M. (2010). Implicit perceptual anticipation triggered by statistical learning. The Journal of Neuroscience, 30, 11177-11187.

Weiss, D. J., Gerfen, C., \& Mitchel, A. D. (2009). Speech segmentation in a simulated bilingual environment: A challenge for statistical learning? Language Learning and Development, 5, 30-49.
Zhao, J., Ngo, N., McKendrick, R., \& Turk-Browne, N. B. (2011). Mutual interference between statistical summary perception and statistical learning. Psychological Science, 22, 1212-1219.

Zhao, J., Al-Aidroos, N., \& Turk-Browne, N. B. (2013). Attention is spontaneously biased toward regularities. Psychological Science, 24, 667-677. 